\title{
Parâmetros populacionais e tabela de vida estática em 2010 para Minas Gerais
}

\section{Population parameters and static life table in 2010 for Minas Gerais}

Daniel Silva Nascimento ${ }^{1 *}$, Ozíres Caroline Dias Privado Santos, Erick William Sandoval, Afonso Pelli

\section{RESUMO}

O objetivo deste estudo foi caracterizar e estimar parâmetros populacionais para a população Mineira em 2010. Para tanto foi construída uma tabela de vida estática. Foram utilizados dados publicados do censo de 2010, pela Coordenação de População e Indicadores Sociais, da Diretoria de Pesquisas, ligada à Gerência de Estudos e Análises da Dinâmica Demográfica do Instituto Brasileiro de Geografia e Estatística. Foram utilizados os dados de número de pessoas morrendo dentro de um intervalo de tempo, a proporção de vivos em cada classe etária, comparando com os nascidos vivos, a mortalidade e sobrevivência específica por classe etária e, a expectativa de vida média, por classe etária. As principais conclusões são que a taxa de sobrevivência permanece relativamente constante ao longo da vida, com redução da mortalidade nas classes etárias mais avançadas. Em 2010, a maior parte da população mineira estava em período produtivo e nasceram mais homens que mulheres. Porém, a taxa de mortalidade específica por classe etária é maior para os homens ao longo de toda a vida; dessa forma, após 25 anos a proporção tende a inverter, aumentando a proporção de mulheres até a senescência e morte.

Palavras-chave: Demografia; Dinâmica populacional; Sudeste Brasileiro; Populações

\section{ABSTRACT}

The aim of this study was to characterize and estimate population parameters for the Minas Gerais population in 2010. For this purpose, a static life table was built. Data published from the 2010 census were used, by the Coordination of Population and Social Indicators, of the Directorate of Research, linked to the Management of Studies and Analysis of Demographic Dynamics of the Brazilian Institute of Geography and Statistics. Data were used on the number of people dying within a time interval, the proportion of living in each age group, comparing with live births, mortality and specific survival by age group, and the average life expectancy by age group. The main conclusions are that the survival rate remains relatively constant throughout life, with a reduction in mortality in older age groups. In 2010, most of the population of Minas Gerais was in a productive period and more men were born than women. However, the age-specific mortality rate is higher for men throughout their lifetime; thus, after 25 years the proportion tends to reverse, increasing the proportion of women until senescence and death.

Keywords: Demography; Population dynamics; Southeastern Brazil; Populations

\footnotetext{
${ }^{1}$ Disciplina de Ecologia \& Evolução. Instituto de Ciências Biológicas e Naturais. Universidade Federal do Triângulo Mineiro *E-mail: danieluftm@ hotmail.com
} 


\section{INTRODUÇÃO}

No passado recente a expectativa de vida das populações tem aumentado progressivamente. Esse aumento da expectativa de vida, específico por classe etária, não é uniforme. As populações apresentam atributos e, dentre esses, a variabilidade intra e interpopulacional desempenham importante papel no cenário. No século 20, no Brasil, de forma semelhante a outros países, houve um aumento de quase 30 anos para a expectativa de vida dos brasileiros. Esse ganho, no entanto, não foi similar para os dois gêneros pois, as mulheres têm maior longevidade. Segundo Veras et al., (1987) além de fatores biológicos, podem existir outros fatores associados ao fenômeno, como questões culturais e sociais.

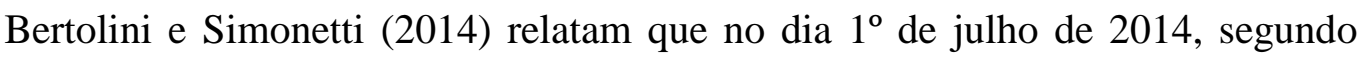
estimativa do Instituto Brasileiro de Geografia e Estatística (IBGE); o Brasil tinha 202,7 milhões de habitantes. A expectativa de vida, no geral, passou de 74,1 em 2011 para 74,6 anos em 2012. Em relação ao sexo, para os homens passou de 70,6 para 71 anos e para as mulheres subiu de 77,7 para 78,3 anos no mesmo período.

As tabelas de vida refletem as relações entre as populações e o meio ambiente. Podem ser construídas por dois princípios diferentes. O primeiro seria acompanhando uma coorte, ou seja, um grupo de organismos que nasceram mais ou menos na mesma época, que não apresentam obrigatoriamente relação de parentesco e que vivem no mesmo ambiente e tempo. As anotações devem ser desde o nascimento do primeiro indivíduo até a morte do último indivíduo. Nesta situação é possível anotar a fecundidade. Esta tabela é denominada de Tabela de Vida Dinâmica (RICKLEFS, RELYEA, 2016; PELLI et al. 2007; SAVORDELLI, PELLI, 2012).

Outra opção seria a Tabela de Vida Estática. Neste caso um recenseamento da população é realizado em determinado instante. Seria como uma "fotografia" da população. Porém, com essa metodologia não é possível anotar a taxa de fecundidade específica por classe etária (PELLI et al. 2007).

O objetivo do estudo foi caracterizar a população Mineira em 2010 e, estimar alguns parâmetros populacionais. Para tanto foi construída uma tabela de vida estática para a população Mineira, separada por gênero, recenseada pelo Instituto Brasileiro de Geografia e Estatística em 2010. 


\section{MATERIAIS E MÉTODOS}

Os dados foram coletados a partir de informações disponibilizadas pelo IBGE em sítio público institucional na rede mundial de computadores, pela Coordenação de População e Indicadores Sociais da Diretoria de Pesquisas, ligada à Gerência de Estudos e Análises da Dinâmica Demográfica (http://www.ibge.gov.br).

$\mathrm{O}$ número de indivíduos em cada faixa etária foi designado por $\mathrm{n}_{\mathrm{x}}$, enquanto a proporção do número de indivíduos vivo em dada classe etária, em função do número de nascidos vivos foi anotado como $1_{\mathrm{x}}$, designado aqui como supervivência. A taxa de mortalidade foi designada como $\mathrm{m}_{\mathrm{x}}$; sendo calculada como o número atual de indivíduos, menos o número de indivíduos na próxima classe etária, dividido pelo número de indivíduos vivos. A sobrevivência, designada aqui por $S_{\mathrm{x}}$, foi calculada pela porcentagem. $\mathrm{L}_{\mathrm{x}}$ e $\mathrm{T}_{\mathrm{x}}$ não apresentam significado biológico, mas são utilizados para o cálculo da expectativa de vida $\left(e_{\mathrm{x}}\right)$. $\mathrm{L}_{\mathrm{x}}$ é o número vivo, em média, de um intervalo de tempo para o próximo intervalo; e $\mathrm{T}_{\mathrm{x}}$ é calculado como a somatória de $\mathrm{L}_{\mathrm{x}}$. A e é calculada como o $\mathrm{T}_{\mathrm{x}}$ sobre o $\mathrm{n}_{\mathrm{x}}$ (PEIXOTO et al., 2016; PELLI et al., 2007).

A curva de sobrevivência foi construída com os dados de supervivência $\left(l_{\mathrm{x}}\right)$ (SAVORDELLI, PELLI, 2014).

\section{RESULTADOS E DISCUSSÃO}

A Tabela 1 apresenta a Tabela de Vida Estática para a população mineira, a partir dos dados do censo do IBGE, em 2010.

Como observado na Figura 1, a taxa de sobrevivência é relativamente constante até a faixa etária 55-59 anos. Após esse intervalo observa-se desaceleração. Os dados indicam que grande parte da população Mineira ultrapassam o período reprodutivo, o que condiz com a transição demográfica relatada por outros autores (CORTEZ et al., 2019).

O período produtivo do brasileiro mediano está entre 14 e 65 anos, embora a formalidade seja alcançada aos 28,6 anos, e apenas $8 \%$ destes trabalham após 60 anos (IBGE, 2010).

Na Figura 2, em azul, está destacado entre 15 e 59 anos, faixas que concentram maior parte da população economicamente ativa. Do total de 142.191 .455 mineiros, 80.301.606 estavam nesse intervalo e 61.889 .849 são economicamente improdutivos. 
Esta figura indica a tendência de relação entre a estrutura etária e o desempenho econômico da sociedade, fato também observado por Stampe, Pozzobon e Niquito (2020).

A Figura 3 apresenta a proporcionalidade entre a expectativa média de vida das mulheres, comparada a dos homens. Como pode-se observar, ao longo da vida, a expectativa média de vida das mulheres é superior após 30 anos, aproximadamente.

Nas fases iniciais da vida, o homem possui maiores taxas de sobrevivência, específica, por classe etária. A transição ocorre aproximadamente aos 25 anos de idade.

Mas a questão seria: Por qual razão homens vivem menos? Ao pesquisar artigos da área humana, percebe-se a necessidade de aplicar uma lente de gênero para a interpretação dos resultados.

Tabela 1 - Tabela de Vida Estática para a população mineira, a partir dos dados do censo do IBGE, em 2010.

\begin{tabular}{ccccccc}
\hline Faixa Etária & $\mathrm{N}^{\circ}$ ind. morrendo & $\mathrm{n}_{\mathrm{x}}$ & $\mathrm{l}_{\mathrm{x}}$ & $\mathrm{m}_{\mathrm{x}}$ & $\mathrm{S}_{\mathrm{x}}$ & $\mathrm{e}_{\mathrm{x}}$ \\
\hline $0-4$ & 1.338 .553 & 19.957 .444 & 1,00 & 0,067 & 0,93 & 6,62 \\
$5-9$ & 1.497 .792 & 18.618 .891 & 0,93 & 0,080 & 0,92 & 6,07 \\
$10-14$ & 1.711 .110 & 17.121 .099 & 0,86 & 0,100 & 0,90 & 5,55 \\
$15-19$ & 1.742 .648 & 15.409 .989 & 0,77 & 0,113 & 0,89 & 5,11 \\
$20-24$ & 1.757 .058 & 13.667 .341 & 0,68 & 0,129 & 0,87 & 4,70 \\
$25-29$ & 1.727 .867 & 11.910 .283 & 0,60 & 0,145 & 0,85 & 4,32 \\
$30-34$ & 1.617 .371 & 10.182 .416 & 0,51 & 0,159 & 0,84 & 3,97 \\
$35-39$ & 1.435 .712 & 8.565 .045 & 0,43 & 0,168 & 0,83 & 3,62 \\
$40-44$ & 1.392 .455 & 7.129 .333 & 0,36 & 0,195 & 0,80 & 3,25 \\
$45-49$ & 1.312 .183 & 5.736 .878 & 0,29 & 0,229 & 0,77 & 2,92 \\
$50-54$ & 1.149 .069 & 4.424 .695 & 0,22 & 0,260 & 0,74 & 2,64 \\
$55-59$ & 933.653 & 3.275 .626 & 0,16 & 0,285 & 0,71 & 2,39 \\
$60-64$ & 725.100 & 2.341 .973 & 0,12 & 0,310 & 0,69 & 2,14 \\
$65-69$ & 549.163 & 1.616 .873 & 0,08 & 0,340 & 0,66 & 1,88 \\
$70-74$ & 431.005 & 1.067 .710 & 0,05 & 0,404 & 0,60 & 1,59 \\
$75-79$ & 302.173 & 636.705 & 0,03 & 0,475 & 0,53 & 1,33 \\
$80-84$ & 190.884 & 334.532 & 0,02 & 0,571 & 0,43 & 1,08 \\
$85-89$ & 92.674 & 143.648 & 0,01 & 0,645 & 0,35 & 0,85 \\
$90+$ & 50.974 & 50.974 & 0,00 & 1,000 & 0,00 & 0,50 \\
\hline
\end{tabular}

Onde: $n_{x}$, número de indivíduos; $1_{x}$, supervivência; $m_{x}$, mortalidade; $S_{x}$, sobrevivência e $e_{x}$, expectativa de vida.

Fonte: dos autores 
Figura 1 - Curva de sobrevivência para a população Mineira, segundo o Censo IGBE de 2010.

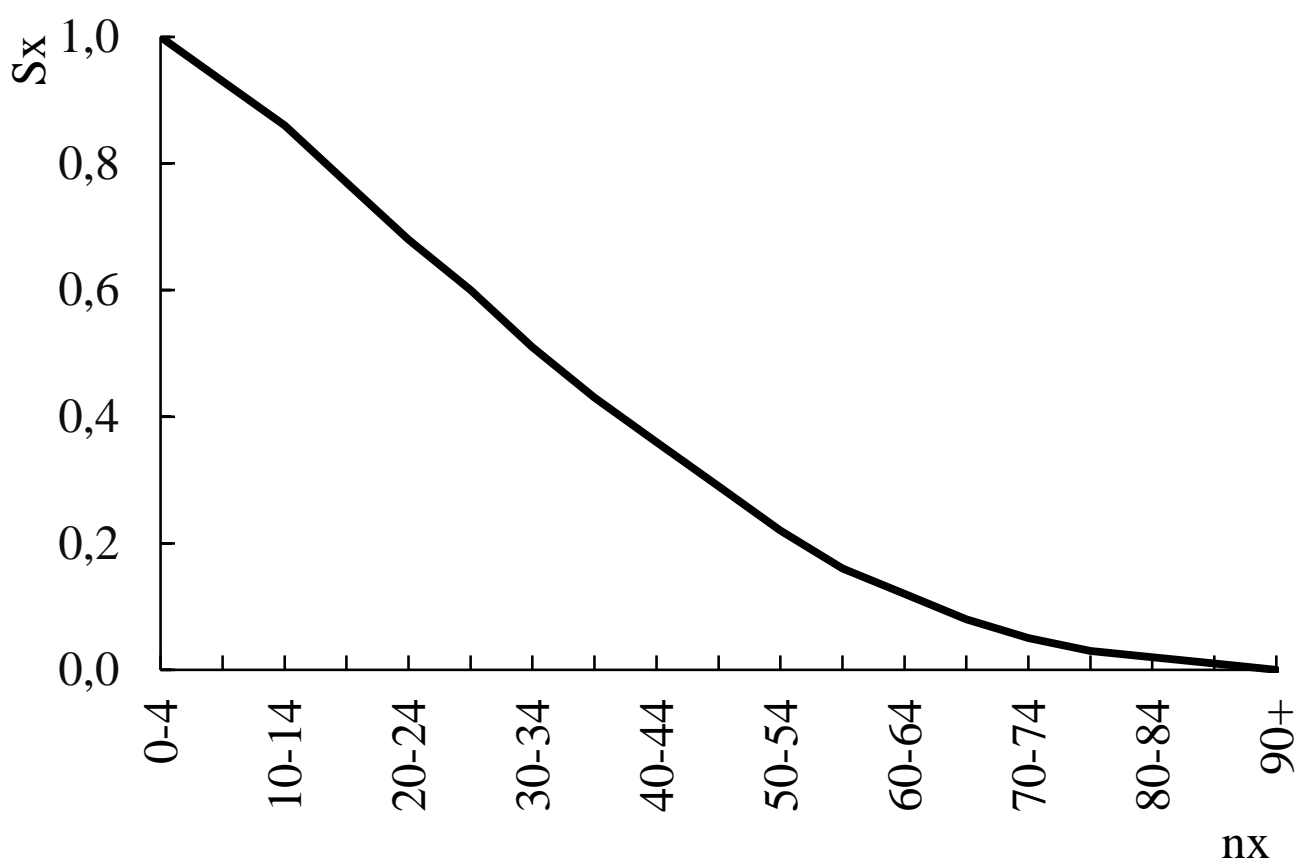

Fonte: dos autores

Figura 2 - Distribuição etária da população Mineira em 2010, com destaque em azul para as classes produtivas, adaptado a partir da Tabela de Vida Estática.

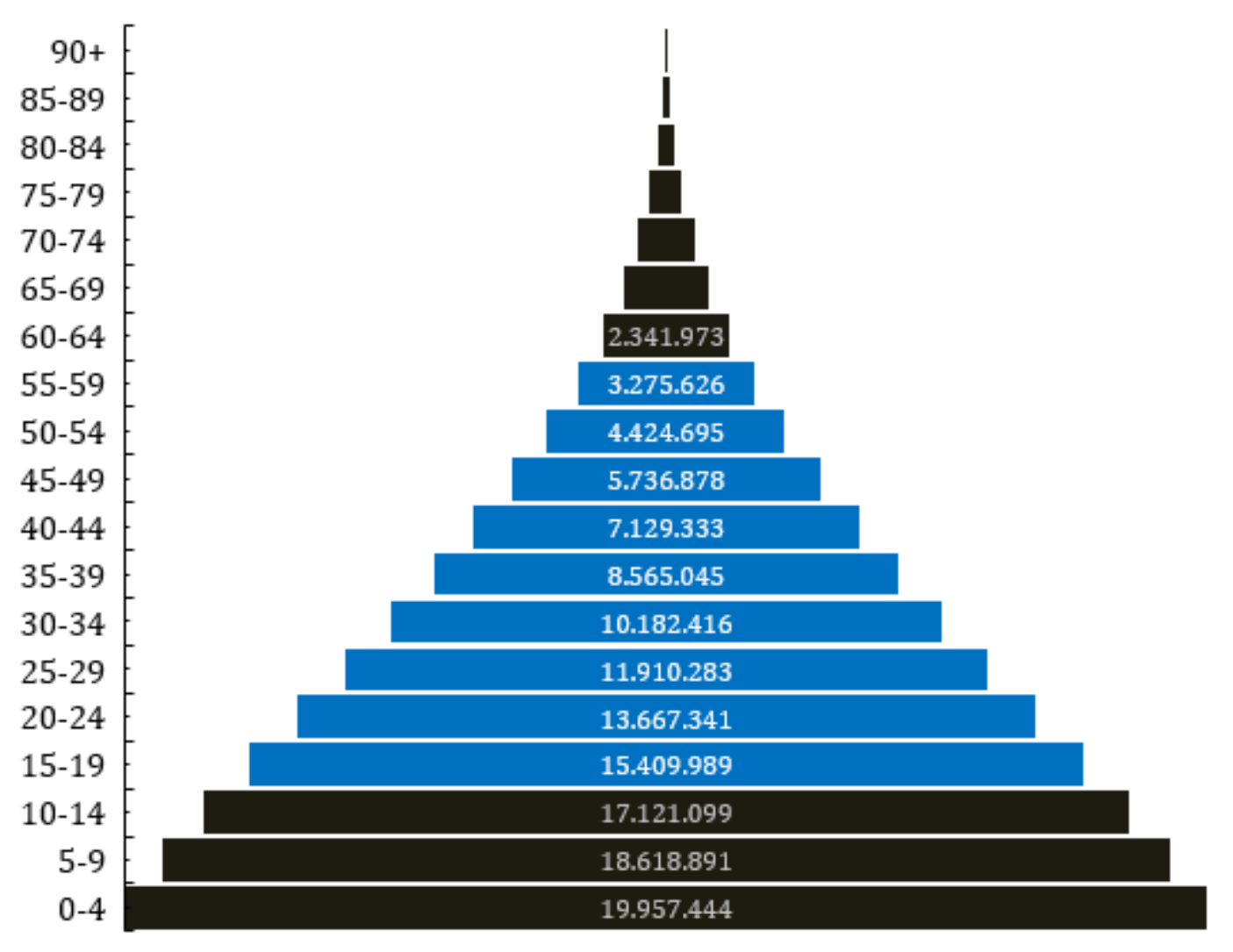

Fonte: dos autores 
Nos países mais desenvolvidos a diferença entre homens e mulheres tende a diminuir um pouco, mas não muito (LAURENTI et al, 2005; OLIVEIRA e MENDES, 2010; VERAS et al, 1987).

Figura 03 - Proporção entre o número de mulheres e homens, para a população Mineira, em 2010, por classe etária.

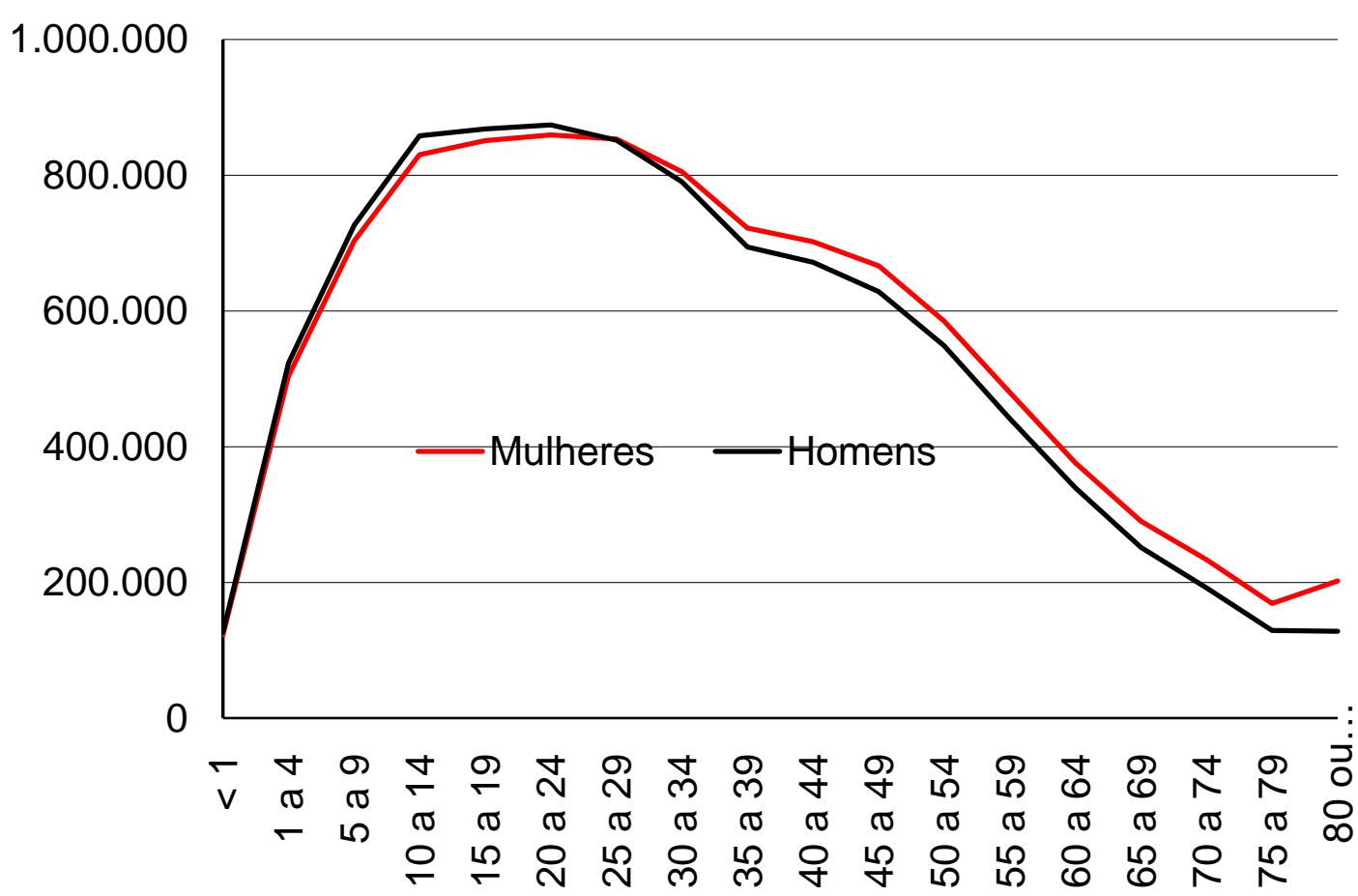

Fonte: dos autores

Segundo Laurenti et al (2005), a menor sobrevida masculina teve e tem, na literatura científica, apenas abordagem biológica. Os autores também apontam que nascem e morrem mais homens em todas as faixas etárias. Embora o fator biológico seja importante nesse contexto, não se pode desconsiderar o aspecto sociocultural nas diferenças dos gêneros, o que por alguns autores é questionável.

Gomes et al, (2007) diz que muitas pesquisas indicam maior número de mortes em homens, com doenças crônicas e negligenciadas se comparados as mulheres. Apesar da morbidade masculina ser superior a feminina, há menor assiduidade de homens no setor de atenção primária à saúde. Esse fato pode ser compreendido na socialização do homem, na relação feita do cuidado a saúde como um comportamento não viril, e isso, geralmente estimula os homens a rejeitar os cuidados com a própria saúde enquanto se encontra saudável ou com enfermidades leves. Entretanto mais uma vez os autores do presente estudo criticam essa abordagem. Segundo dados do IBGE, a expectativa de vida média de homens é bem menor que mulheres, em todas as classes etárias, incluindo aqui 
todos os anos desde o nascimento. Mesmo com os mesmos genitores, na mesma família, nas classes etárias de 0 a 1; 1 a 2; e assim sucessivamente, a expectativa de vida média dos homens é menor; logo, não podemos atribuir ao próprio cuidado, mas sim a fragilidade do gênero masculino, frente aos fatores do ambiente, o que muitas vezes é confundido com força física.

Muitas vezes ouve-se dizer que a expectativa de vida do homem é Y anos, e da mulher $\mathrm{X}$ anos, sendo $\mathrm{X}=\mathrm{Y}+\mathrm{Z}$. Porém, quando se observa a Tabela 1, percebe-se que a expectativa de vida, é um atributo distintivo para cada grupo etário (ou específico da classe etária), e que valores médios não têm significado biológico. Para os indivíduos que estão na classe etária entre 25 e 29 anos, a expectativa de vida média, em 2010, era de 4,32; ou seja, esses indivíduos ainda vão viver, em média, mais 4,32 intervalos de tempo. Já que as classes etárias foram plotadas de cinco em cinco anos eles viveriam em média, mais 21,6 anos. Ou, após 4,32 intervalos de tempo, metade da população atual (11.910.283) irá morrer. Ou seja, no intervalo de tempo 45-49 anos, apenas 5.736.878 estão vivos, o que é uma boa aproximação do valor calculado $(50 \%$ de $11.910 .283=$ 5.955.141,5). O valor não é exato, pois consideramos o intervalo de 4, e não 4,32.

A vantagem feminina na sobrevida é um fenômeno de característica biológica, social e comportamental. Existem vários fatores, citados por Oliveira e Mendes, os quais comprovam maior resiliência feminina (OLIVEIRA, MENDES, 2010; ESKES, HAANEN, 2005; MELO et al, 2008).

Quando considerados hábitos de vida, presença de doenças crônicas no Brasil em todas as regiões, ocorreu crescimento da prevalência de hábitos nocivos, como: sedentarismo, sobrepeso, hipertensão arterial, insuficiente atividade física no tempo livre e diabetes (VERAS et al, 1987).

Essa postura mais matizada, adotada nessa abordagem, permitirá perceber nuances entre gêneros antes não percebidas; tornando-se necessária para garantir a percepção da complexidade entre gêneros, com vistas à saúde pública.

Distinguir mulheres e homens como grupos-alvo levará a melhorias, uma vez que ambos são diferentes em muitos aspectos e, portanto, requerem abordagens distintas (DEMEO, WEISS, 2009).

Apesar do conhecimento específico de gênero e sexo na área da biologia, quando se pondera homens versus mulheres, muitas diferenças são nítidas, como taxa metabólica, sistema respiratório e circulatório; tônus muscular e habilidades específicas. Porém, 
algumas distinções permanecem não muito evidentes. Como a epidemiologia da asma, que é mais prevalente em meninos que meninas. Essa tendência inverte e, após a puberdade, ou pouco mais tarde, próximo aos 20 anos, o risco para mulheres é maior em países europeus (DEMEO, WEISS, 2009).

O sexo precisa ser incluído na pesquisa biológica básica; com base em abordagens baseadas em hipóteses (e não apenas no empirismo) e por pesquisadores dedicados. O delineamento experimental necessita de estudos clínicos e epidemiológicos. A sutileza necessária para a pesquisa de gênero deve ser reconhecida como uma dimensão relevante na epidemiologia. Deve-se estar ciente de que as diferenças de gênero resultam mais de condições biológicas, que genéticas, do ambiente, epigenética e mesmo das condições sociais e culturais.

O presente estudo abre diversas possibilidades para a abordagem populacional, não apenas no Estado de Minas Gerais, mas bem provavelmente na concepção das políticas públicas para as populações. Lembrando assim, sempre, da importância do levantamento censitário, realizado pelo Estado.

\section{REFERÊNCIAS}

BERTOLINI, D N P; SIMONETTI, J. P. O gênero masculino e os cuidados de saúde: a experiência de homens de um centro de saúde. Esc. Anna Nery, 18(4): 722-727. http://dx.doi.org/10.5935/1414-8145.20140103. 2014.

CORTEZ, A. C. L.; SILVA, C. R. L.; SILVA, R. C. L.; DANTAS, E. H. M. Aspectos gerais sobre a transição demográfica e epidemiológica da população brasileira. Enferm Bras, 18(5);700-9. https://doi.org/10.33233/eb.v18i5.2785. 2019.

ESKES, T., HAANEN, C. Why do women live longer than men? European Journal of Obstetrics \& Gynecology and Reproductive Biology, 133 (2): 126-133. doi.org/10.1016/j.ejogrb.2007.01.006. 2005.

GOMES, R.; NASCIMENTO, E. F.; ARAUJO, F. C. Por que os homens buscam menos os serviços de saúde do que as mulheres? As explicações de homens com baixa escolaridade e homens com ensino superior. Cad. Saúde Pública, 23 (3): 565-574. doi.org/10.1590/S0102-311X2007000300015. 2007.

IBGE - INSTITUTO BRASILEIRO DE GEOGRAFIA E ESTATÍSTICA. Sinopse do censo demográfico: 2010. Rio de Janeiro, 2011. Disponível em: http://www.ibge.gov.br/populacao/projecao. Acesso em: 07 de jan. de 2021. 
IBGE - INSTITUTO BRASILEIRO DE GEOGRAFIA E ESTATÍSTICA. Diretoria de Pesquisas (DPE), Coordenação de População e Indicadores Sociais. Disponível em: FTP://ftp.ibge.gov.br/tabuas_completas_de_mortalidade_2016. Acesso em: 22 de Novembro de 2020.

LAURENTI, R.; JORGE, M. H. P. M.; GOTLIEB, S. L. D. Perfil epidemiológico da morbi-mortalidade masculina. Ciênc. saúde coletiva 10 (1): 35-46. https://doi.org/10.1590/S1413-81232005000100010. 2005.

MElO, E. M.; CÔRTES, M. C. J. W.; MIRANDA, P. S. C.; CÂMARA, A. C. S.; ALVES, R. A.; PEREIRA, V. O. M.; BRANCO, Â. S. C. C. Eles morrem mais do que elas. Por quê? Rev Med Minas Gerais. 18(4): S12-S18. 2008.

OLIVEIRA, I. T.; MENDES, M. F. A diferença de esperança de vida entre homens e mulheres: Portugal de 1940 a 2007. Anál. Social, XLV (194): 115-138. 2010.

PEIXOTO, P. G.; OLIVEIRA, R. V.; MIRANDA, V. S.; PAULINO, T. P.; ANDRADE, R. M.; PELLI, A. Avaliação proteica e parâmetros populacionais de Cynaeus angustus Le Conte (Coleoptera: Tenebrionidae). EntomoBrasilis, 9: 108 - 113. 2016.

PELLI, A.; SILVA, M. A.; SARMENTO, F. R.; MARTINS, E.; MATA, S. A.; DOMINGUES, M. A.; RAMIREZ, L. E. Parâmetros populacionais para Triatoma sordida Stal, 1859, o vetor mais frequente da doença de Chagas no Triângulo Mineiro (Heteroptera, Triatominae). Revista da Sociedade Brasileira de Medicina Tropical, 40 (1): 25-28, https://doi.org/10.1590/S0037-86822007000100005. 2007.

PEIXOTO, P. G. OLIVEIRA, R. V. MIRANDA, V. S. PAULINO, T. P. ANDRADE, R. M. PELLI, A. Avaliação proteica e parâmetros populacionais de Cynaeus angustus Le Conte (Coleoptera: Tenebrionidae). EntomoBrasilis, 9(2): 108-113. 2016.

RICKLEFS, R. RELYEA, R. A economia da natureza. 7. ed. ed. Rio de Janeiro, Guanabara Koogan, 636 p. 2016.

SAVORDELLI, N. C.; PELLI, A. Dados preliminares sobre a tabela de vida estática de Dermestes maculatus DeGeer, 1774 em Condições de Laboratório. Brazilian Journal of Forensic Sciences, Medical Law and Bioethics, 1: (3): 77-83, https://doi.org/10.17063/bjfs1(3)y201277. 2012.

SAVORDELLI, N. C.; PELLI, A. Tabela de vida estática de Dermestes maculatus Degeer, 1774 em condições de laboratório. Revista SODEBRAS, 9: 96 - 99. 2014.

STAMPE, M. Z; POZZOBON, F.; NIQUITO, T. W. Impactos da estrutura etária sobre o desempenho econômico das regiões do Brasil entre 1991 e 2010. R. bras. Est. Pop., 37: 1-25, e0116, http://dx.doi.org/10.20947/S0102-3098a0116. 2020. 
VERAS, R. P.; RAMOS, L. R.; KALACHE, A. Crescimento da população idosa no Brasil: transformações e consequências na sociedade. Rev. Saúde Pública, São Paulo, 21(3): 225-233. https://doi.org/10.1590/S0034-89101987000300007. 1987.

Recebido em: 01/09/2021

Aprovado em: 20/09/2021

Publicado em: 30/092021 